Ann. Zootech., 1987, 36 (2), 153-170

\title{
Influence de la complémentation des foins traités à l'ammoniac sur leur valeur nutritive
}

\author{
H. BENAHMED, J.P. DULPHY
}

avec la collaboration technique de Madeleine DUDILIEU, Marie JAILLER, Jacqueline JAMOT, H. BOUSQUET et L. L'HOTELIER

INRA, Laboratoire des Aliments, Centre de Recherches de Clermont-Ferrand-Theix F 63122 Ceyrat

\section{Résumé}

Nous avons mis en place 3 essais sur moutons. Dans le premier, un foin humide non traité ou traité avec 8 et $16 \mathrm{~kg}$ d'ammoniac par tonne a été étudié. Cet essai a confirmé l'effet négatif du traitement à l'ammoniac sur l'activité cellulolytique dans le rumen mais pas sur la valeur azotée du foin lorsqu'il est distribué seul aux animaux.

Dans un second essai, nous avons distribué un foin non traité, un foin traité et deux rations mixtes ( $2 / 3$ foin non traité $+1 / 3$ foin traité ; $1 / 3$ foin non traité $+2 / 3$ foin traité). Le foin non traité était pauvre en azote et le traitement a très légèrement amélioré sa valeur azotée. Mais là encore, le traitement a diminué l'activité cellulolytique dans le rumen.

Dans un troisième essai, le foin traité du second essai a été distribué avec des compléments devant améliorer l'activité cellulotytique dans le rumen (tourteau de soja, minéraux, iso-acides). Avec ces compléments la valeur azotée du foin traité s'est légèrement améliorée. Il en a été de même pour l'activité cellulolytique du rumen.

La dilution du foin traité par un foin non traité ainsi que la complémentation améliorent l'activité cellulolytique dans le rumen. Néanmoins, la digestibilité in sacco du foin traité reste bien en deçà de sa digestibilité potentielle, mesurée sur des animaux ingérant du foin de luzerne. Par ailleurs l'amélioration de la valeur azotée des foins traités semble possible par une complémentation adéquate.

Nous avons mesuré le $\mathrm{pH}$ et les teneurs en ammoniac du jus de rumen dans les 3 essais et les teneurs en AGV dans le troisième essai. azotée.

Mots clés: Foin, traitement à l'ammoniac, complémentation, valeur alimentaire, rétention

\section{Introduction}

Une série d'essais sur le traitement des foins à l'ammoniac (BEnAHMED \& DulPHY, 1985 et 1986) a montré que la valeur azotée de ces foins traités était, en générral, plus faible que celle des foins témoins non traités. Deux causes principales peuvent être avancées : la destruction par le traitement de certains acides aminés du fourrage et la baisse de l'activité cellulolytique dans le rumen. Il semble bien, en effet, qu'une partie des acides aminés du fourrage soit détruite par le traitement (NELson et al., 1985 ; LALIGANT, 1986) ce qui doit diminuer la quantité de protéines alimentaires arrivant dans l'intestin grêle. Par ailleurs, l'activité microbienne du rumen, et donc la synthèse des 
protéines microbiennes, paraît aussi réduite par le traitement à l'ammoniac (NeLson et al., 1985) comme il l'est par le traitement à la soude (Tisserand, Bellet \& Masson, 1986). Nous avions déjà mis en évidence ce phénomène en étudiant l'activité cellulolytique dans le rumen de moutons recevant des pailles traitées (Dulphy, KomaR \& ZWAENEPOEL, 1984). Ce phénomène renforce très probablement la baisse de valeur azotée que nous avons constatée.

Nous n'avons pas cherché à agir sur la première cause de cette baisse car elle semble presque impossible à éviter, en particulier pour les foins humides (ZWAENEPOEL, 1986). Par contre, il devrait être possible d'améliorer l'activité microbienne dans le rumen. Nous avons essayé d'y parvenir de deux façons : (1) en "diluant » le foin traité dans la ration, (2) en rajoutant au foin traité des facteurs de croissance pour les microbes (protéines, minéraux, iso-acides). Nous avons supposé, en effet, que la faible activité microbienne observée dans le rumen pouvait être due tout d'abord à une présence trop importante de composés phénoliques libérés par le traitement et toxiques pour les microbes (Jung \& FAHEY, 1981 ; Gouet et al., 1986). Nous avons présumé ensuite que la destruction d'acides aminés gênait l'activité de ces microbes soit directement, par l'absence de certains de ces acides, soit indirectement, par l'absence de support carboné particulier pour la synthèse d'acides aminés. Nous avons, dans ce but, apporté des iso-acides dont Hemsley \& Molr, 1963 ; Maeng \& Baldwin, 1976 ; Tuah \& TAIT, 1985 avaient montré l'importance.

Nous avons, d'une part, estimé l'activité microbienne en suivant la digestion de substrats cellulosiques mis en sachets de nylon dans le rumen de moutons consommant les foins étudiés et d'autre part, estimé les effets sur la valeur azotée à travers des bilans azotés (BenAhmed \& Dulphy, 1986).

\section{Matériel et méthodes}

Avant de mettre en place deux essais visant à optimiser une activité cellulolytique dans le rumen nous avons d'abord apprécié cette activité (essai I) pour 3 foins, non traité ou traités, distribués seuls, afin de confirmer nos premières observations (BENAHMED \& DULPhy, 1985). Dans l'essai II, nous avons comparé la distribution simultanée d'un foin non traité et d'un foin traité sec, en proportions différentes dans la ration. Enfin, dans l'essai III, nous avons distribué différents compléments avec ce même foin traité.

\section{A. Fourrages (tabl. 1)}

L'essai I a été réalisé avec le foin $\mathrm{F}$ de qualité moyenne (Benahmed \& Dulphy, 1986), foin humide (26\% d'humidité), traité en juin 1984 avec 0 (F1), 8 (F2T) et $16 \mathrm{~kg}$ (F3T) d'ammoniac par tonne de matière brute selon le procédé Armako au CEMAGREF de Montoldre (ZWAENEPOEL, 1986). Juste après l'injection, la température des foins traités est montée à $60^{\circ} \mathrm{C}(\mathrm{F} 2 \mathrm{~T})$ et $70^{\circ} \mathrm{C}$ (F3T) pendant quelques heures, puis s'est maintenue vers $30^{\circ} \mathrm{C}$ pendant 10 jours. Les meules de foins traités ont été ouvertes 13 semaines après l'injection d'ammoniac et les foins ont été maintenus tels quels 3 semaines, puis hachés et distribués à des moutons. 
TABLEAU 1

Composition chimique des fourrages utilisés.

Chemical composition of the different diets.

\begin{tabular}{|c|c|c|c|c|c|c|}
\hline \multirow[b]{2}{*}{$\begin{array}{l}\text { Essai } \\
\text { Trial }\end{array}$} & \multirow[b]{2}{*}{$\begin{array}{l}\text { Fourrage } \\
\text { Forage }\end{array}$} & \multirow{2}{*}{\multicolumn{2}{|c|}{$\begin{array}{l}\text { Teneurs en MS (\%) } \\
\text { lors de la distribution } \\
D M(\%) \text { at feeding }\end{array}$}} & \multicolumn{3}{|c|}{$\begin{array}{l}\text { Teneurs en } \mathrm{g} / \mathrm{kg} \text { MS } \\
\text { Contents in } \mathrm{g} / \mathrm{kg} \mathrm{DM}\end{array}$} \\
\hline & & & & Cendres & $\begin{array}{l}\text { Matières } \\
\text { azotées }\end{array}$ & $\begin{array}{c}\text { Cellulose } \\
\text { brute }\end{array}$ \\
\hline \multirow{3}{*}{ I } & $\begin{array}{l}\text { Foin non traité } \ldots \ldots \ldots \\
\text { Untreated hay }\end{array}$ & $\mathrm{F} 1$ & 85 & 69 & 87 & 305 \\
\hline & $\begin{array}{l}\text { Foin traité } 8 \mathrm{~kg} \mathrm{NH}_{3} / \mathrm{t} \ldots \\
\text { Treated hay }\end{array}$ & $\mathrm{F} 2 \mathrm{~T}$ & 83 & 70 & 134 & 314 \\
\hline & $\begin{array}{l}\text { Foin traité } 16 \mathrm{~kg} \mathrm{NH}_{3} / \mathrm{t} \ldots \\
\text { Treated hay }\end{array}$ & F3T & 82 & 75 & 167 & 317 \\
\hline \multirow{2}{*}{ II et III } & $\begin{array}{l}\text { Foin non traité } \ldots \ldots \ldots \\
\text { Untreated hay }\end{array}$ & K1 & 89 & 77 & 50 & 364 \\
\hline & $\begin{array}{l}\text { Foin traité } 30 \mathrm{~kg} \mathrm{NH}_{3} / \mathrm{t} \ldots \\
\text { Treated hay }\end{array}$ & $\mathrm{K} 2 \mathrm{~T}$ & 87 & 81 & 113 & 361 \\
\hline
\end{tabular}

Pour les essais II et III, nous avons traité, en juin 1985, $1500 \mathrm{~kg}$ d'un foin sec de fétuque (K) de mauvaise qualité avec $3 \%$ d'ammoniac. Le tas a été débâché 14 semaines plus tard, laissé en l'état 2 semaines pour libérer l'ammoniac en excès ; le foin a été ensuite haché et distribué à des moutons. Du foin témoin non traité (K1) avait été conservé par ailleurs.

\section{B. Animaux}

Les moutons utilisés pour les mesures de digestibilité et de bilans azotés étaient des béliers castrés de race Texel, âgés de 18 mois environ et pesant $48 \mathrm{~kg}$ (essais I et II) et $45 \mathrm{~kg}$ (essai III). Ils étaient maintenus en cage à métabolisme permettant la collecte totale des fèces et de l'urine.

Nous avons utilisé par ailleurs des animaux fistulisés du rumen, âgés de 2 à 3 ans et pesant de 50 à $60 \mathrm{~kg}$, pour mesurer les principaux paramètres de la digestion dans le rumen.

\section{Schémas expérimentaux}

Chacun des essais a été conduit selon un schéma en carré latin : 2 moutons $\times 3$ foins $\times 3$ périodes pour l'essai I, 2 moutons $\times 4$ rations $\times 4$ périodes pour les essais II et III, soit au total 6 moutons d'une part, 8 de l'autre. 


\section{TABLEAU 2}

Caractéristiques des régimes étudiés.

Characteristics of the different diets.

\begin{tabular}{|c|c|}
\hline $\begin{array}{l}\text { Essai I : (ad libitum) } \\
\text { Trial I }\end{array}$ & $\begin{array}{l}1 \text { - Foin non traité }(\mathrm{F} 1) \\
\text { Untreated hay } \\
2 \text { - Foin traité } 8 \mathrm{~kg} \mathrm{NH}_{3} / \mathrm{t}(\mathrm{F} 2 \mathrm{~T}) \\
\mathrm{NH}_{3} \text { treated hay } \\
3 \text { - Foin traité } 16 \mathrm{~kg} \mathrm{NH}_{3} / \mathrm{t}(\mathrm{F} 3 \mathrm{~T}) \\
\mathrm{NH}_{3} \text { treated hay }\end{array}$ \\
\hline 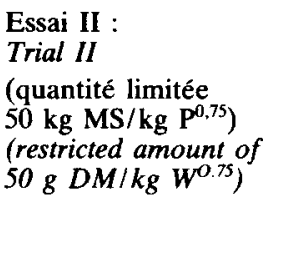 & $\begin{array}{l}1-100 \% \text { foin non traité }(\mathrm{K} 1) \\
100 \% \text { untreated hay } \\
2-66 \% \text { foin non traité }+33 \% \text { foin traité } \\
66 \% \text { untreated hay }+33 \% \text { treated hay } \\
3-33 \% \text { foin non traité }+66 \% \text { foin traité } \\
33 \% \text { untreated hay }+66 \% \text { treated hay } \\
4-100 \% \text { foin traité }(\mathrm{K} 2 \mathrm{~T}) \\
100 \% \text { treated hay }\end{array}$ \\
\hline $\begin{array}{l}\text { Essai III : } \\
\text { Trial III } \\
\text { (quantité limitée } 50 \mathrm{~g} \\
\text { MS } / \mathrm{kg} \mathrm{P} \mathbf{P}^{0,75} \text { ) } \\
\text { (restricted amount of } \\
50 \mathrm{~g} \mathrm{DM} / \mathrm{kg} \mathrm{W}^{0.75} \text { ) }\end{array}$ & 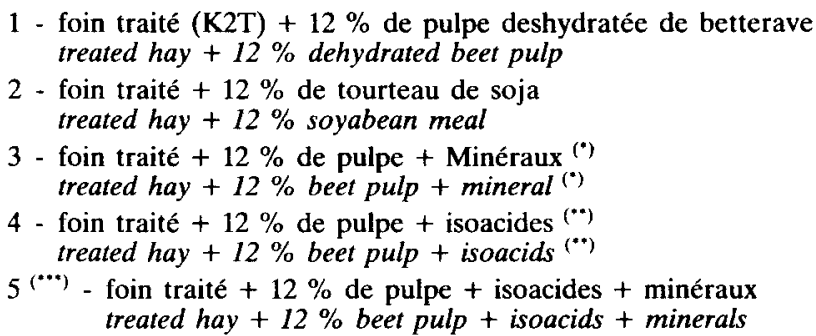 \\
\hline
\end{tabular}

(') Minéraux : $20 \mathrm{~g}$ d'un mélange spécial par $\mathrm{kg}$ de ration contenant : $8 \%$ de sulfate de soude, $9 \%$ de sulfate de magnésie, $52 \%$ de phosphate bicalcique, $26 \%$ de sel marin, $3 \%$ de soufre en fleur, $0,094 \%$ de sulfate de cuivre, $0,95 \%$ de sulfate de zinc, $0,002 \%$ de sulfate de cobalt, $0,48 \%$ de sulfate de manganèse, $0,48 \%$ de sulfate de fer, $0,003 \%$ d'iodure de calcium et $0,001 \%$ de selenite de sodium.

Minerals : $20 \mathrm{~g} / \mathrm{kg}$ of diet including : $8 \%$ soda sufate, $9 \%$ magnesia sulfate, $52 \%$ bicalcic phosphate, $26 \%$ sodium salt, $3 \%$ flower sulphur, $0.094 \%$ copper sulfate, $0.95 \%$ zinc sulfate, $0.002 \%$ cobalt sulfate, $0.48 \% \mathrm{Mn}$ sulfate, $0.48 \%$ iron sulfate, $0.003 \%$ calcium iodide and $0.001 \%$ sodium selenite.

$\left({ }^{* *}\right)$ Isoacides : $3 \mathrm{~g}$ d'acide isovalérique $+2,3 \mathrm{~g}$ d'acide isobutyrique par $\mathrm{kg}$ brut de ration.

Isoacids : $3 \mathrm{~g}$ isovaletic acid $+2.3 \mathrm{~g}$ isobutyric acid $/ \mathrm{kg}$ diet.

$\left({ }^{* * *}\right)$ Sur fistulés seulement.

Only in fistulated animals.

Les moutons fistulisés du rumen (2 par essai) ont reçu successivement les différentes rations étudiées. Celles-ci sont décrites dans le tableau 2. Ces rations ont toujours été distribuées pendant des périodes de 3 semaines, les mesures étant effectuées les 6 derniers jours de chaque période.

Dans l'essai I, les foins ont été offerts à volonté (10\% de refus), mais, pour ne pas avoir de biais dû à des niveaux d'alimentation différents, toutes les rations des essais II et III ont été distribuées en quantité limitée (50 g de $\left.\mathrm{MS} / \mathrm{kg} \mathrm{P}^{0,75}\right)$. Dans tous les cas, les animaux ont reçu 2 repas par jour à $8 \mathrm{~h}$ et à $16 \mathrm{~h}$. 


\section{Mesures}

Nous avons effectué les mesures suivantes :

- quantités ingérées (différence entre quantités offertes et refusées) en gramme ;

- digestibilité des régimes (matière organique et matières azotées) par collecte totale des fèces (digestibilité en \%);

- rétention azotée pour chaque régime (Benahmed \& Dulphy, 1986) en gramme d'azote ;

- activité cellulolytique : 4 sachets de nylon contenant un même substrat par essai, placés dans le rumen de 2 moutons recevant les régimes expérimentaux (pendant 8,24 et 48 h) (Demarquilly \& Chenost, 1969);

- cinétique de digestion des foins : 4 sachets de nylon contenant les foins expérimentaux eux-mêmes, placés dans le rumen de 2 moutons recevant un foin de luzerne ;

- caractéristiques du jus de rumen pour chaque régime expérimental : $\mathrm{pH}, \mathrm{N}$ $\mathrm{NH}_{3}$, AGV (Jouany, 1981) : à 8, 9, 10,11, 12, 14 et 16 h durant 2 jours consécutifs sur les 2 moutons fistulés recevant les régimes expérimentaux.

\section{E. Analyses}

La préparation des échantillons et les analyses effectuées ont été les mêmes que dans nos études précédentes (Benahmed \& Dulphy, 1985 et 1986).

\section{Résultats}

\section{A. Quantités ingérées (tabl. 3)}

Dans l'essai I, les traitements n'ont pas amélioré significativement les quantités volontairement ingérées par les moutons $(+8,3 \%$ seulement pour le foin traité avec 16 $\mathrm{kg} / \mathrm{t}$ d'ammoniac).

Dans les essais II et III, bien que les quantités totales offertes aient été limitées à un même niveau, les animaux ont fait quelques refus surtout pour les régimes 1 et 2 de l'essai II, très pauvres en azote.

\section{B. Digestibilité de la matière organique (tabl. 3)}

Dans l'essai I, on n'a pas noté d'amélioration significative de la digestibilité par le traitement à l'ammoniac, les doses utilisées étant probablement trop faibles. Dans l'essai II, par contre, il y a eu une augmentation significative de la digestibilité après le traitement ( +7 points) et déjà un accroissement très net avec $33 \%$ de foin traité dans la ration, lequel est probablement dû surtout à l'apport d'azote au foin témoin carencé, car après traitement, la digestibilité enzymatique (Aufrere, 1982) n'augmente que de 2,2 points. Enfin, dans l'essai III, où l'apport d'azote n'était théoriquement pas limitant, il y a eu peu de différence entre les digestibilités du foin selon les traitements, 


\section{TABLEAU 3}

Quantités ingérées et digestibilités des régimes étudiés. Intake and digestibility of the different diets.

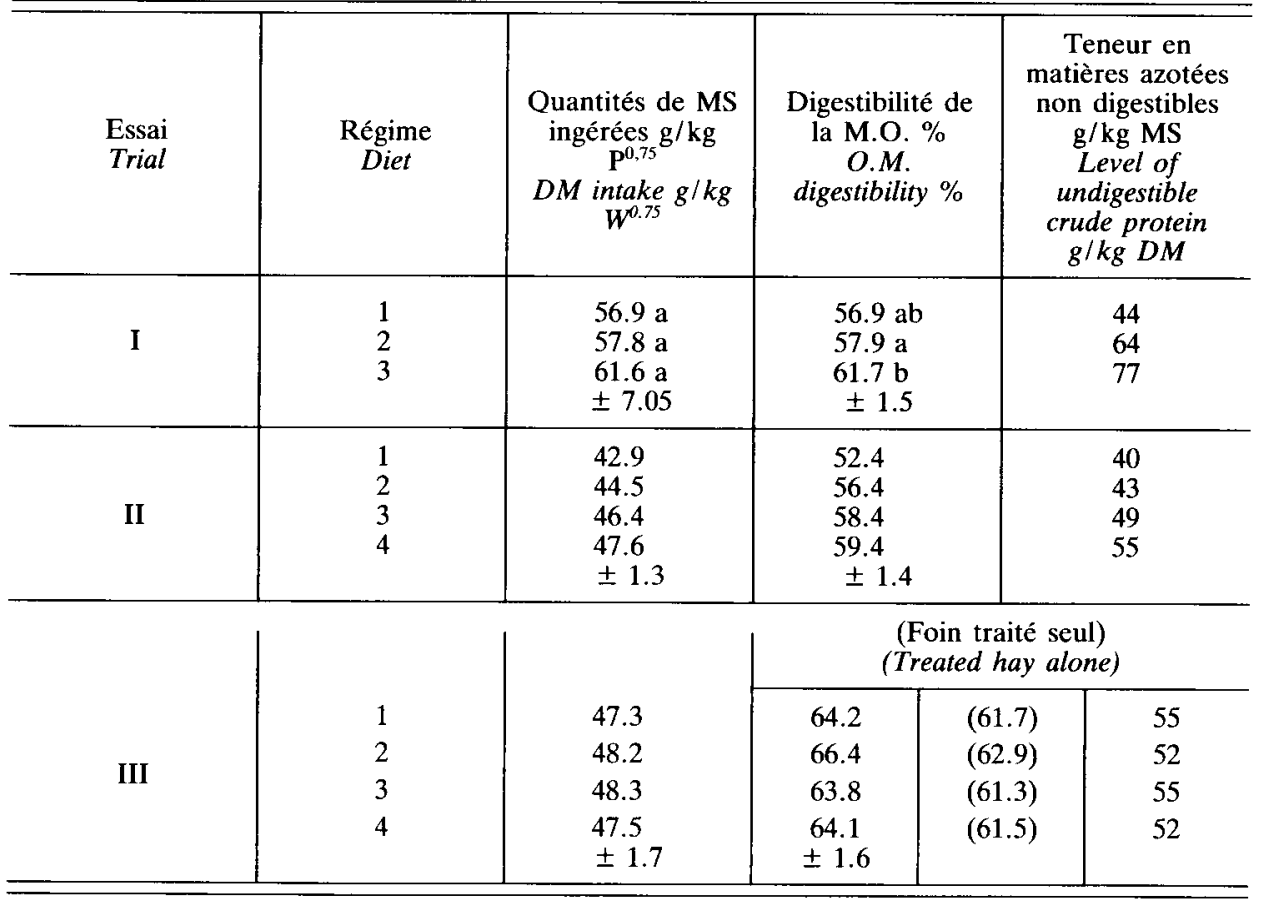

sauf une tendance à une digestibilité plus élevée $(+1,4$ point $)$ en présence de tourteau de soja.

\section{Rétention azotée (tabl. 4)}

Dans l'essai I, pour des apports d'azote très différents (de 15,3 à $30,5 \mathrm{~g} /$ jour mais de 15,3 à 16,6 provenant du fourrage lui-même), le traitement a augmenté la rétention azotée, quoique non significativement. Elle a été la meilleure lorsque la dose d'ammoniac utilisée était faible, alors que la quantité de matière organique digestible ingérée ne variait pas. Par contre, elle a eu tendance à moins augmenter avec la dose d'ammoniac la plus élevée, alors que la quantité de matière organique digestible ingérée augmentait de $12 \%$.

Dans l'essai II, les rétentions azotées n'ont pas varié significativement avec les régimes, la rétention la plus faible étant observée avec le foin non traité distribué seul, lequel était mal ingéré.

Enfin dans l'essai III, l'apport de minéraux ou d'iso-acides n'a pas davantage amélioré significativement les bilans azotés qui ont cependant été meilleurs avec 
TABLeaU 4

Bilans azotés (g/animal/jour).

Nitrogen retention for the different diets (g/animal/day).

\begin{tabular}{|c|c|c|c|c|c|}
\hline $\begin{array}{l}\text { Essai } \\
\text { Trial }\end{array}$ & $\begin{array}{c}\text { Régime } \\
\text { Diet }\end{array}$ & $\begin{array}{l}\mathrm{N} \text { ingéré } \\
\text { Ingested } N\end{array}$ & $\begin{array}{c}\mathrm{N} \text { fécal } \\
\text { Excreted } N\end{array}$ & $\begin{array}{l}\mathrm{N} \text { urinaire } \\
\text { Urinary } N\end{array}$ & $\begin{array}{l}\mathrm{N} \text { retenu } \\
\text { Retained } N\end{array}$ \\
\hline $\mathbf{I}$ & $\begin{array}{l}1 \\
2 \\
3\end{array}$ & $\begin{array}{l}15.3 \\
23.4 \\
30.5\end{array}$ & $\begin{array}{r}7.4 \\
10.9 \\
13.7\end{array}$ & $\begin{array}{r}7.2 \\
10.0 \\
15.0\end{array}$ & $\begin{array}{l}+0.68 \mathrm{a} \\
+2.49 \mathrm{a} \\
+1.79 \mathrm{a} \\
( \pm 1.5)\end{array}$ \\
\hline II & $\begin{array}{l}1 \\
2 \\
3 \\
4\end{array}$ & $\begin{array}{r}6.7 \\
9.4 \\
12.7 \\
16.0\end{array}$ & $\begin{array}{l}5.0 \\
5.6 \\
6.8 \\
7.6\end{array}$ & $\begin{array}{l}3.6 \\
4.9 \\
7.0 \\
9.1\end{array}$ & $\begin{array}{l}-1.86 \mathrm{a} \\
-1.16 \mathrm{a} \\
-1.10 \mathrm{a} \\
-0.81 \mathrm{a} \\
( \pm 1.24)\end{array}$ \\
\hline III & $\begin{array}{l}1 \\
2 \\
3 \\
4\end{array}$ & $\begin{array}{l}15.2 \\
22.5 \\
15.3 \\
15.1\end{array}$ & $\begin{array}{l}7.0 \\
6.9 \\
7.3 \\
7.0\end{array}$ & $\begin{array}{r}7.6 \\
12.6 \\
7.1 \\
7.1\end{array}$ & $\begin{array}{l}+0.56 \mathrm{a} \\
+2.87 \mathrm{~b} \\
+0.97 \mathrm{a} \\
+0.84 \mathrm{a} \\
( \pm 0.73)\end{array}$ \\
\hline
\end{tabular}

l'apport de tourteau, ce qui est logique. On peut par ailleurs calculer, pour les régimes étudiés, une valeur azotée approximative en PDIE d'après les propositions de BENAHMED \& DULPHY (1986) :

$$
\text { PDI ing. }=36,3+0,474 \text { P.V. }+1,538 \text { MAT ret. }
$$

Cela conduit, pour l'essai II, aux valeurs respectives de $53,59,57$ et $59 \mathrm{~g}$ de PDIE/kg MS de fourrage ingéré. Pour l'essai III, on obtient respectivement : 77, 105, 80 et $80 \mathrm{~g} / \mathrm{kg}$ de $\mathrm{MS}$ de ration, soit $74,80,78$ et 77 pour le foin seul. La complémentation semble donc avoir très nettement amélioré la valeur azotée du foin, bien que les essais II et III aient été réalisés avec des animaux différents.

\section{Caractéristiques du jus de rumen}

1. $p H$ (tabl. 5)

Dans l'essai I, le pH moyen du jus de rumen a baissé après le traitement à $0,8 \%$ d'ammoniac $(-0,15)$, mais a augmenté avec le traitement à $1,6 \%(+0,14)$ par rapport au foin non traité.

Dans l'essai II, le fait d'introduire du foin traité dans les rations a, globalement, augmenté le $\mathrm{pH}(+0,13)$, les différences entre les régimes 2,3 et 4 étant faibles.

Enfin dans l'essai III (fig. 1), le pH a été plus élevé avec les régimes expérimentaux qu'avec le régime 1 sans additif particulier, un pH élevé ayant été observé pour le régime foin + pulpe + iso-acides. 


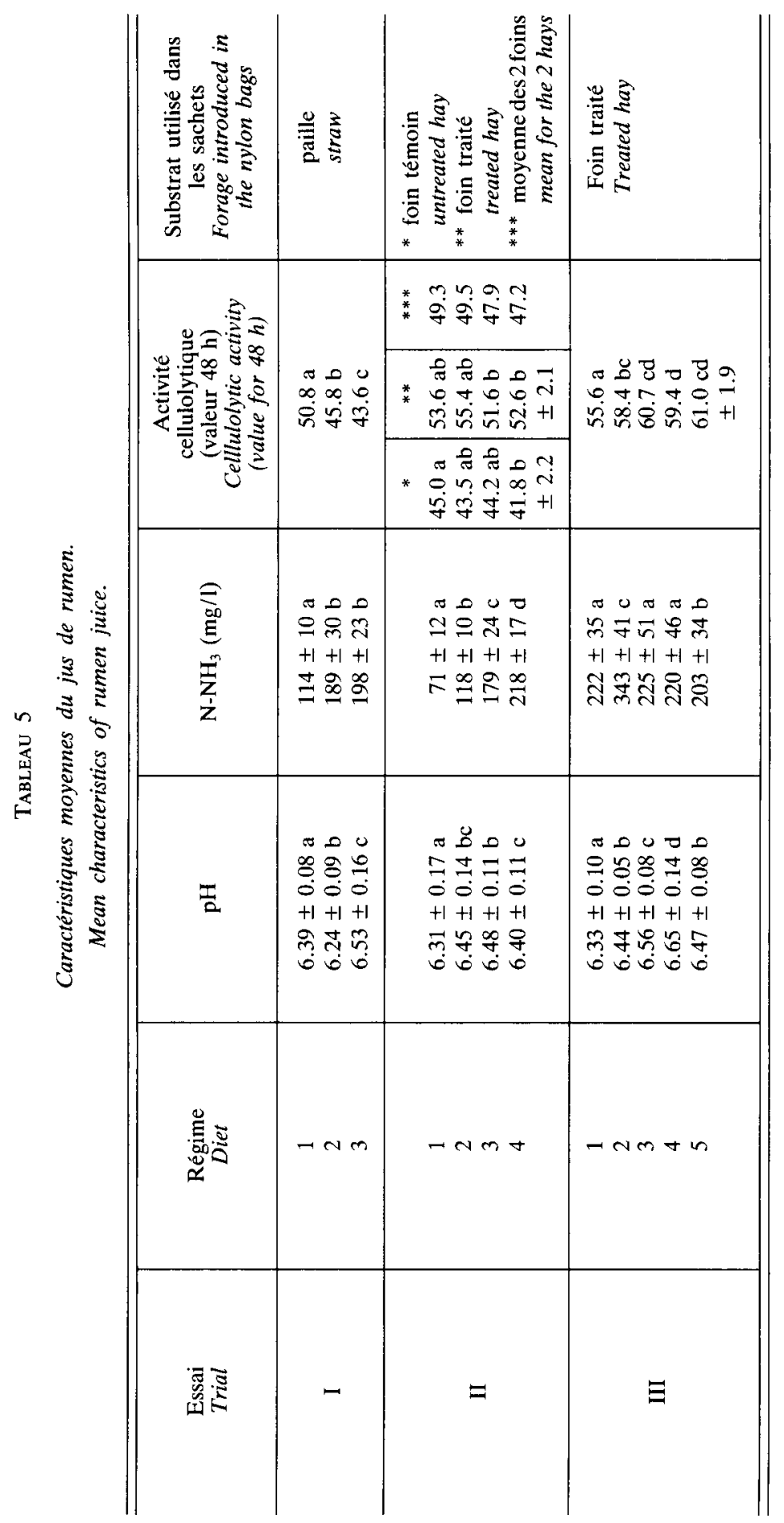




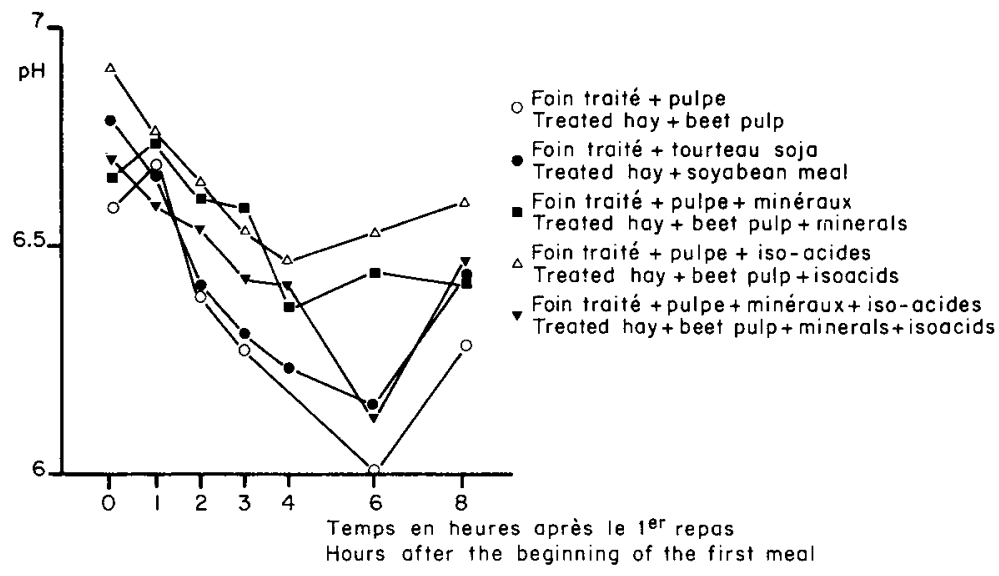

FIG. 1

Evolution du $p H$ du jus de rumen dans l'essai III.

Variation in the $p H$ of rumen juice in trial III.

\section{2. $\mathrm{NH}_{3}($ tabl. 5)}

Dans l'essai I (fig. 2), les teneurs en azote ammoniacal du jus de rumen ont été fortement accrues par le traitement, mais les valeurs pour le foin témoin ont toujours été supérieures à $60 \mathrm{mg} / \mathrm{l}$.

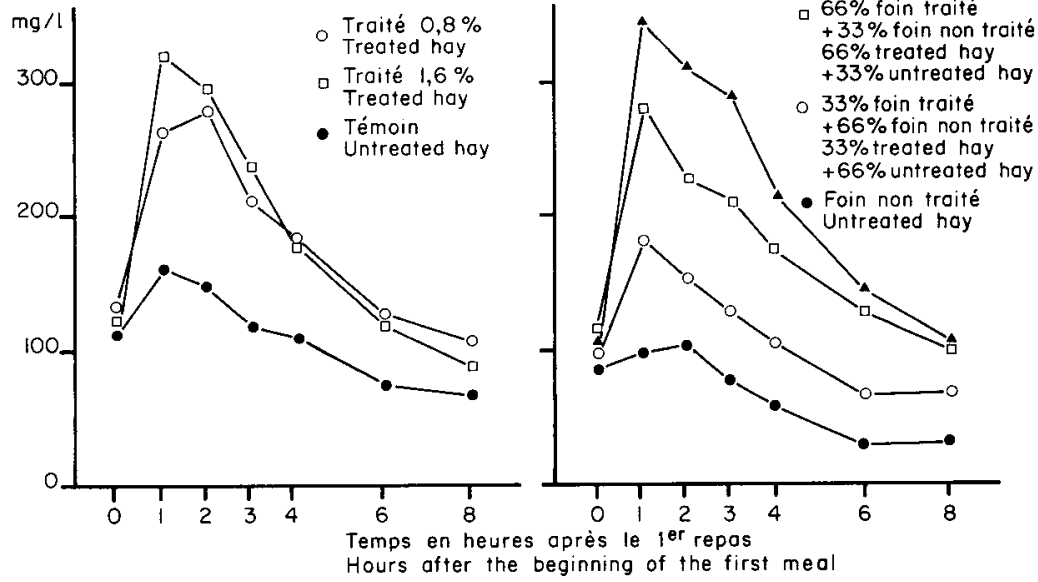

FIG. 2

Evolution des teneurs en $\mathrm{N}-\mathrm{NH}_{3}$ dans le jus de rumen.

Variation in $\mathrm{N}-\mathrm{NH}_{3}$ levels in rumen juice.

A gauche: Essai 1; on the left Trial I.

$A$ droite: Essai 2; on the right Trial II. 
Dans l'essai II (fig. 2), le foin témoin étant carencé en azote, les teneurs en azote ammoniacal du jus de rumen ont été faibles et sont passées au-dessous de $50 \mathrm{mg} / 1$, 6 heures après le début du repas. Les valeurs moyennes ont ensuite nettement augmenté au fur et à mesure que la part de foin traité dans la ration devenait plus importante.

Enfin dans l'essai III (fig. 3) les valeurs obtenues pour le foin traité complémenté avec pulpe (régimes 1, 3, 4 et 5) ont été en moyenne de $218 \mathrm{mg} / 1$, valeur identique à celle du foin traité seul de l'essai II. Il y a eu peu de différence entre les 4 régimes cités, mais une forte augmentation lors de l'apport de tourteau de soja.

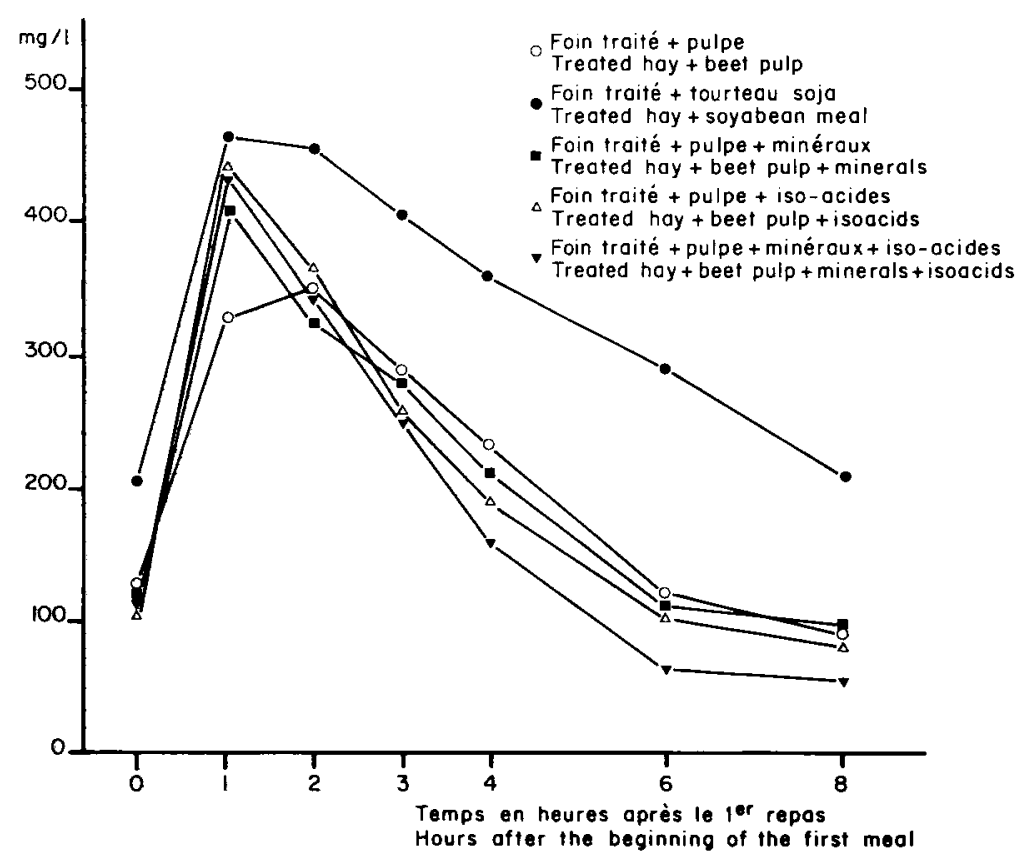

FIG. 3

Evolution des teneurs en $\mathrm{N}-\mathrm{NH}_{3}$ du jus de rumen dans l'essai III. Variation in $\mathrm{N}-\mathrm{NH}_{3}$ levels in rumen juice in trial III.

\section{Activité cellulolytique (tabl. 5)}

L'activité cellulolytique a été estimée dans l'essai I par la cinétique de dégradation d'un substrat identique (paille) pour les 3 régimes; la digestibilité in sacco de ce substrat a diminué significativement avec le traitement à l'ammoniac (fig. 4). Nous pouvons donc considérer qu'il en a été de même pour l'activité cellulolytique dans le rumen. 


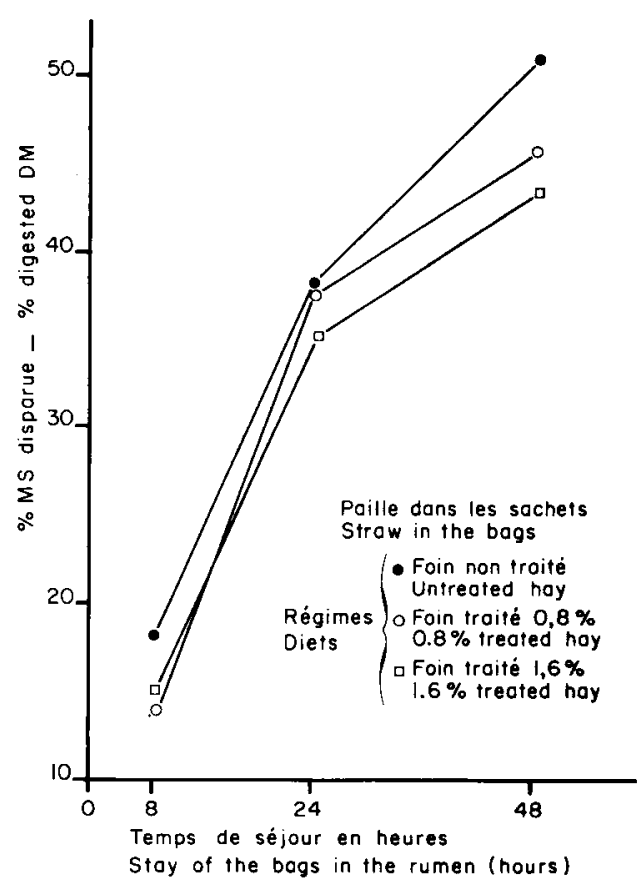

Fig. 4

Activité cellulolytique induite dans l'esssai 1.

Cellulolytic activity induced in trial $I$.

Dans l'essai II, nous avons utilisé comme substrat les 2 foins expérimentaux. Dans ce cas également, en moyenne, l'activité cellulolytique a été diminuée par le traitement, mais assez faiblement (fig. 5).

Enfin dans l'essai III (fig. 6), le fait de complémenter avec du tourteau ou de distribuer des minéraux et des iso-acides avec la pulpe a augmenté significativement l'activité cellulolytique. La comparaison entre les essais II et III, dans lesquels nous avons utilisé le même substrat, indique clairement les effets positifs des combinaisons choisies, les meilleures se rapprochant de ce qui est observé dans le rumen de moutons ingérant du foin de luzerne (fig. 5).

\section{E. Cinétique et digestion des foins (tabl. 6)}

La cinétique de digestion dans le rumen des foins non traités et traités, dans les essais I et II, a été mesurée de 2 façons : avec des moutons ingérant du foin de luzerne ou avec ceux ingérant le foin expérimental lui-même (cf. activité cellulolytique). Dans l'essai I, le traitement à l'ammoniac a eu une action positive, significative $(+5$ points environ à $48 \mathrm{~h}$, contre seulement +2 points in vitro). Cette action a été beaucoup plus 


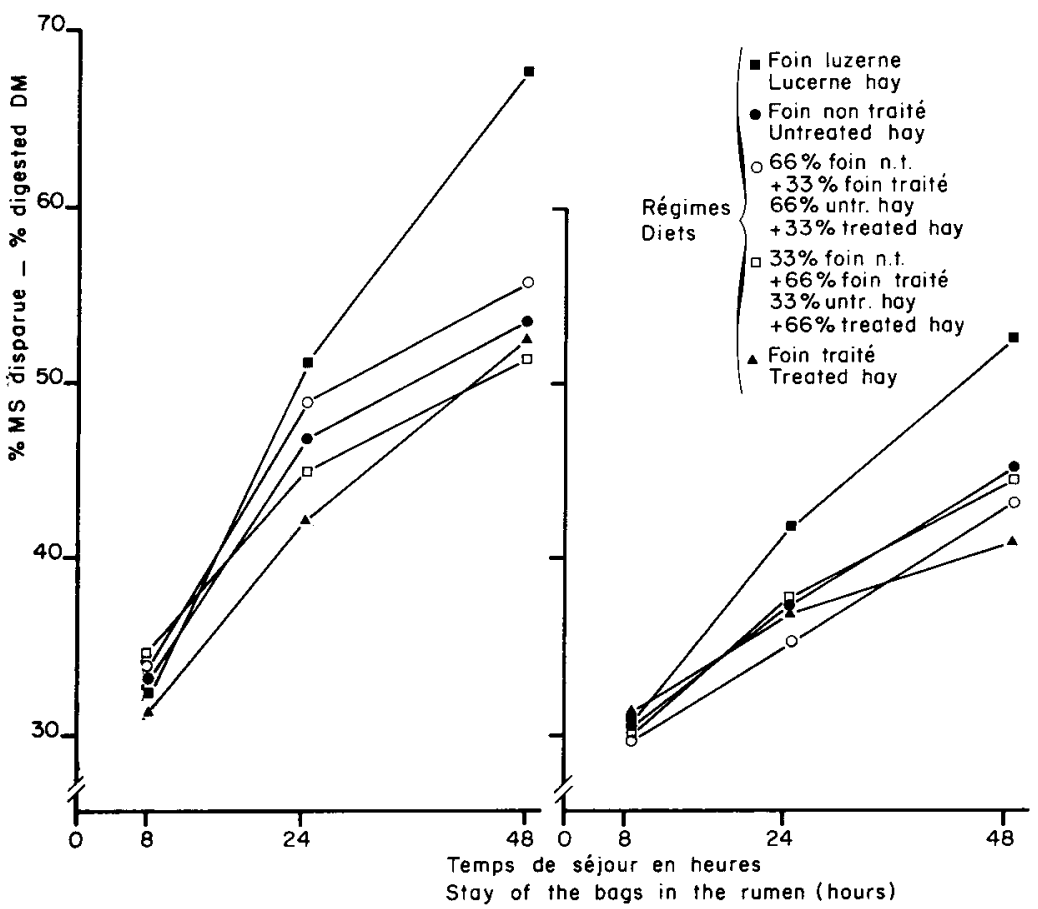

Fig. 5

Activité cellulolytique induite dans l'essai II.

Cellulolytic activity induced in trial 1.

A gauche: Foin traité dans les sachets; on the left: Treated hay in the bags. $A$ droite : Foin non traité dans les sachets; on the right: Untreated hay in the bags.

\section{TABleaU 6}

Digestibilité in sacco des foins expérimentaux à $48 \mathrm{~h}$.

In sacco digestibility of experimental hays after $48 \mathrm{~h}$ stay in rumen.

\begin{tabular}{c|c|c|c}
\hline \hline Essai & Fourrage \\
Trial & Forage & $\begin{array}{c}\text { Moutons ingérant } \\
\text { du foin de luzerne } \\
\text { Sheep fed with lucerne } \\
\text { hay }\end{array}$ & $\begin{array}{c}\text { Moutons ingérant } \\
\text { le même foin } \\
\text { Sheep fed with } \\
\text { the experimental hay }\end{array}$ \\
\hline & F1 & 61.6 & 58.7 \\
I & F2T & 64.1 & 61.0 \\
& F3T & 66.7 & 63.4 \\
& K1 & \pm 1.0 & \pm 1.5 \\
\hline & K2T & 52.6 & 45.0 \\
& II & 67.5 & 52.6 \\
& & \pm 1.6 & \pm 2.3 \\
\hline \hline
\end{tabular}




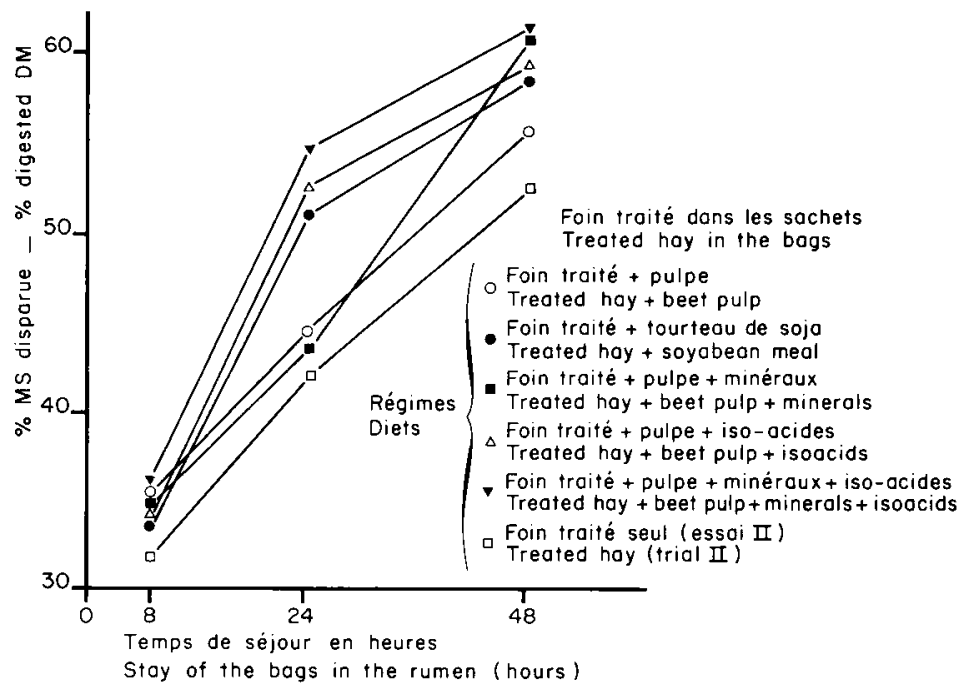

FIG. 6

Activité cellulolytique induite dans l'essai III.

Cellulolytic activity induced in trial III.

nette dans l'essai II, surtout en présence du foin de luzerne : +15 points dans ce cas contre +7 points in vivo. Notons la différence considérable qui existe entre les valeurs obtenues pour le foin traité lorsque les sachets sont placés dans le rumen de moutons recevant du foin traité $(52,6$ à $48 \mathrm{~h})$ ou du foin de luzerne $(67,5$ à $48 \mathrm{~h})$.

\section{F. Orientation des fermentations dans le rumen (tabl. 7)}

Nous n'avons mesuré l'orientation des fermentations dans le rumen que dans l'essai III. La teneur en AGV totaux a été assez constante pour les différents régimes sauf, curieusement, pour celui avec minéraux et iso-acides ( 75 contre en moyenne 88 pour les autres). Par ailleurs, bien que beaucoup de valeurs soient significativement différentes, l'orientation générale des fermentations a été à peu près la même pour tous les régimes, ce qui est normal, puisque tous comportaient $88 \%$ du même foin. Il existe cependant quelques différences de détail : une proportion plus élevée d'acide acétique pour le régime foin + pulpe + minéraux, moins d'acide propionique pour le régime foin + tourteau, plus d'acide isobutyrique pour les régimes avec iso-acides, plus d'acide iso-valérique également pour ces deux régimes, mais aussi en présence de tourteau. Enfin, il y a eu plus d'acides valérique et caproïque, toujours en présence de tourteau. Le régime foin + tourteau se distingue donc par la présence notable d'acides gras provenant de la dégradation d'acides aminés. 


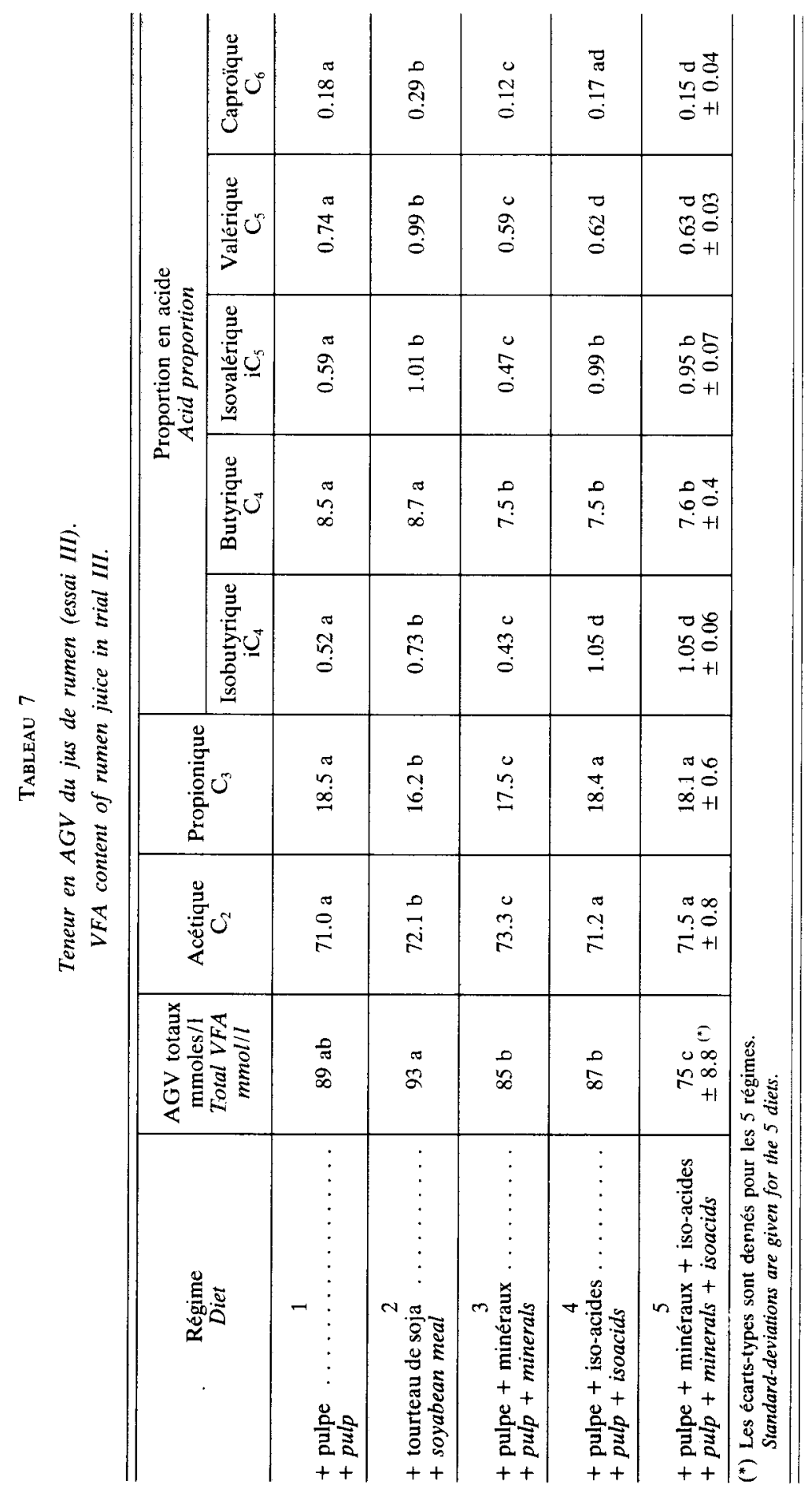




\section{Discussion-Conclusion}

Dans ces essais, nous avons utilisé deux foins très différents, l'un ayant une valeur azotée moyenne (essai I) et l'autre une valeur azotée faible (essai II). Les effets du traitement des foins à l'ammoniac sont semblables à ceux déjà observés (BENAHMED \& DulPHY, 1986): légère amélioration des quantités ingérées et de la digestibilité, qui s'explique avant tout par une vitesse de digestion accrue, notable dans l'essai I, très nette dans l'essai II. On constate cependant un écart important entre les digestibilités in sacco du foin traité de l'essai II, selon le régime de l'animal. Il apparaît, en effet, que les digestibilités in sacco du foin traité sont bien plus faibles que leurs valeurs potentielles lorsque l'animal consomme du foin traité. Ceci confirme la mauvaise activité cellulolytique induite par les foins traités. Néanmoins, ces foins présentent une valeur alimentaire plus élevée que celles des foins non traités, mais probablement plus faible que la valeur potentielle permise par le traitement.

Si on veut tirer le meilleur parti de cette valeur potentielle il faut donc agir sur la composition de la ration : nous avons étudié deux techniques, la dilution du foin traité par du foin non traité et la complémentation par des suppléments stimulant l'activité cellulolytique. Rappelons que nous observons des problèmes identiques lors du traitement à la soude (Dulphy et al., 1982a). Pour améliorer la digestibilité des pailles traitées à la soude nous avions adopté différentes techniques: la limitation de la quantité totale d'aliments distribués qui avait été efficace, le fractionnement de l'apport de concentré, inefficace (Dulphy \& Bienaime, 1983), et surtout le remplacement des concentrés à base d'amidon par des concentrés à base de parois digestibles (DuLPHY et al., 1982b) qui s'était révélé la technique la plus favorable à l'activité cellulolytique.

Dans les essais rapportés dans ce texte, la dilution a eu un effet favorable sur la digestibilité puisque les régimes 2 et 3 de l'essai II ont présenté une digestibilité légèrement supérieure à celle attendue (respectivement $+1,7$ et $+1,3$ point). La complémentation par de la pulpe de betterave et surtout du tourteau de soja a eu également un effet positif sur la digestibilité du foin traité (respectivement $+2,1$ points et $+3,5$ points). Ces améliorations passent par une amélioration globale de l'activité cellulolytique, soit lorsqu'on ajoute du foin non traité dans la ration (essai II), soit lorsqu'on apporte des compléments (essai III). Dans l'essai III, il n'y a cependant pas concordance entre l'amélioration de l'activité cellulolytique, très nette en présence d'isoacides, et l'absence d'augmentation de digestibilité in vivo entre le régime complémenté avec la pulpe seule et la pulpe + iso-acides. Par contre, lorsqu'on remplace la pulpe par du soja il y a à la fois amélioration de l'activité cellulolytique et de la digestibilité in vivo.

Il serait intéressant de connaître plus précisément les causes de la mauvaise activité cellulolytique induite par les fourrages traités à l'ammoniac. Plusieurs sont possibles et peuvent d'ailleurs agir ensemble. On sait que les traitements aux alcalis libèrent des composés phénoliques toxiques pour certaines souches de microorganismes (JuNG \& FAHEY, 1981 ; AKIN, 1982 ; VAREL \& JUNG, 1986). Le traitement doit aussi détruire certains acides aminés indispensables (Nelson et al., 1985; LALIGANT, 1986). La dilution des composés phénoliques (essai II) réduit logiquement leur effet, sans qu'on puisse savoir s'il y a eu un seuil à ne pas dépasser. L'apport de tourteau de soja semble efficace pour compenser les effets dégradants de l'ammoniac sur les acides aminés. Les minéraux ont eu une légère action sur l'activité cellulolytique, mais pas sur la digestibilité in vivo. Il en a été de même des iso-acides très utiles pourtant pour la synthèse de 
protéines bactériennes (Bryant \& Doetsch, 1955 ; Hemsley \& MoIR, 1963 ; Mir, Mir \& Robertson, 1986). Dans nos essais, minéraux et iso-acides ont donc un rôle limité. Il est vrai que nous avons étudié des foins et non des pailles, l'intérêt de ces suppléments étant en principe plus important pour les fourrages très carencés en minéraux et acides aminés.

Il ne semble pas que dans nos essais le $\mathrm{pH}$ du jus de rumen ait joué un rôle important, car il a peu varié selon les régimes. Les teneurs en ammoniac dans le rumen ont, en général, été supérieures à la valeur seuil de $50 \mathrm{mg} / \mathrm{l}$ (INRA, 1978), sauf pour le foin non traité de l'essai II ; leurs maxima ont été modérés dans les essais I et II. Les valeurs élevées trouvées dans l'essai III ont été observées durant un temps court et n'ont probablement pas eu d'effets négatifs importants. Enfin, les teneurs en AGV et les proportions obtenues apportent peu de renseignements sur les variations de valeur nutritive.

Une autre composante importante de la valeur nutritive est la valeur azotée. Nous en avons déjà discuté dans un article précédent (Benahmed \& Dulphy, 1986) dans lequel les résultats de rétentions azotées étaient déjà donnés pour l'essai I et partiellement pour l'essai II. Dans l'essai I, le traitement n'a pas diminué la valeur azotée, il y a eu augmentation dans l'essai II où le foin témoin était carencé en azote. Dans cet essai II, on ne peut cependant pas savoir si la dilution du foin traité a eu un effet positif, compte tenu de l'imprécision de la méthode d'appréciation utilisée. Dans l'essai III, le fait d'apporter $12 \%$ de pulpe dans la ration a eu un effet positif, mais nous ne pouvons pas séparer l'effet de l'apport de protéines par la pulpe d'un effet éventuel sur une meilleure synthèse bactérienne dans le rumen via une meilleure activité cellulolytique. Là encore, l'effet de l'apport de minéraux et celui d'iso-acides est négligeable. L'effet du tourteau de soja est élevé et compréhensible, mais nous sommes, comme pour la pulpe, dans l'impossibilité de savoir précisément si l'amélioration de l'activité cellulolytique a pu jouer un rôle. Les calculs faits, en attribuant à la pulpe et au tourteau la valeur PDIE des Tables (INRA, 1978), indiqueraient bien un effet très positif des compléments : $21 \mathrm{~g}$ pour le tourteau, $18 \mathrm{~g}$ de PDIE pour les autres compléments, mais une grande prudence s'impose dans l'utilisation du résultat de ces calculs.

En conclusion, la différence importante qui existe entre la valeur nutritive potentielle d'un foin traité à l'ammoniac et sa valeur réelle, lorsqu'il est distribué seul, peut être réduite. Nous confirmons donc l'hypothèse que nous avions précédemment formulée (Benahmed \& Dulphy, 1986). Il en résulte que les foins traités ne devraient pas constituer le seul fourrage d'une ration et qu'ils doivent être complémentés, comme les pailles d'ailleurs (CHENOST, 1987), afin de favoriser leur digestion ruminale.

Sur un plan plus général, la décision de traiter un foin devra cependant être mûrement réfléchie car elle est coûteuse et pas toujours adaptée, par exemple pour des animaux à faibles besoins. Cette approche, plus technique et économique du problème, pourra être faite à partir d'essais réalisés sur bovins avec des foins secs (DulPHy et al., 1986a) ou des foins humides (DulPhy et al., 1986b) traités à l'ammoniac. Les résultats présentés ici confirment que le traitement et le mode de distribution doivent être pris en compte en même temps.

Reçu en décembre 1986.

Accepté en avril 1987. 


\section{Summary \\ Supplementation of ammonia-treated hay : effect on the nutritive value}

Three trials were conducted in sheep. In trial I a wet hay was used untreated or treated by 8 and $16 \mathrm{~kg}$ ammonia per ton. In trial II an untreated hay, a treated hay and two combinations of these two hays were fed to sheep. In trial III the same treated hay supplemented with soyabean meal, isoacids and minerals was fed. Supplementation improved cellulolytic activity in the rumen. Chemical composition of hays is given in table 1 and the characteristics of the studied diets in table 2.

In trial $\mathrm{I}$, the treatment increased intake and digestibility (table 3 ). It was the same in trial II where forage offered was limited to $50 \mathrm{~g} \mathrm{DM} / \mathrm{kg} \mathrm{W}^{8.75}$. In trial III supplementation had a positive effect.

Nitrogen retention was measured for all diets (table 4). N-retention increased slightly, but not significantly, after treatment in trial I, and also in trial II. In trial III supplementation had a positive effect. It was concluded that nitrogen value was increased by the treatment. Accordingly, the decrease in nitrogen value observed by other authors does not seem to be systematic, especially when hay has a poor value.

Mean characteristics of rumen juice were measured in the three trials and are given in table 5 . Variation in $\mathrm{pH}$ in trial III is given in figure $1, \mathrm{~N}^{-\mathrm{NH}_{3}}$ in the three trials in figure 2 and figure 3. The maximum level observed was $450 \mathrm{mg} / \mathrm{l}, 1$ hour after the beginning of the first meal.

Cellulolytic activity, estimated by the degradation of straw or hay in nylon bags suspended in the rumen is also given in table 5. A more precise description is given in figure 4 for trial $\mathrm{I}$, in figure 5 for trial III and in figure 6 for trial III. All these figures confirm the negative effect of treatment on cellulolytic activity in the rumen.

«Dilution " of treated hay into the diet (trial II) or addition of adequate complements (trial III) increased cellulolytic activity in the rumen. Nevertheless in sacco digestibility of treated hay never reached potential in sacco digestibility measured in sheep fed lucerne hay (table 6).

VFA content of rumen juice was measured in trial III.

Key words : Hay, ammonia treatment, supplementation feeding value, nitrogen retention.

\section{Références bibliographiques}

AKIN D.E., 1982. Forage cellwall degradation and p-coumaric, ferulic and sinapic acids. Agron. J., 74, 424-428.

Aufrère J., 1982. Etude de la prévision de la digestibilité des fourrages par une méthode enzymatique. Ann. Zootech., 31, 111-130.

Benahmed H., Dulphy J.P., 1985. Note sur la valeur azotée de fourrages pauvres traités par l'urée ou l'ammoniac. Ann. Zootech., 34, 335-346.

Benahmed H., Dulphy J.P., 1986. Influence du traitement des foins à l'ammoniac sur leur valeur azotée appréciée par la méthode des bilans azotés. Ann. Zootech., 35, 387-400.

Bryant M.P., Doetsch R.N., 1955. Factors necessary for the growth of bacteroides succinogenes in the volatile acid fraction of rumen fluid. J. Dairy Sci., 38, 340-350.

Chenost M., 1987. Influence de la complémentation sur la valeur alimentaire et l'utilisation des mauvais foins et des pailles par le ruminant, in "Les fourrages secs : récolte, traitement, utilisation », $16^{\mathrm{c}}$ journées du Grenier de Theix, 21-23 mai 1985, INRA publ., Route de SaintCyr, 78000 Versailles. 
Dermarquilly C., Chenost M., 1969. Etude de la digestion des fourrages dans le rumen par la méthode des sachets de nylon. Liaisons avec la valeur alimentaire. Ann. Zootech., 18, 419436.

Dulphy J.P., Agabriel J., Garel J.P., Giraud J.M., Rouel J., 1986a. Influence du traitement à l'ammoniac des foins tardifs sur leur valeur alimentaire: étude sur génisses et vaches allaitantes. Bull. Tech. CRZV Theix, INRA, 63, 25-30.

Dulphy J.P., Bienaime A., 1983. Etude de la valeur alimentaire des pailles de céréales traitées ou non à la soude. IV - Influence du nombre de distributions de l'aliment concentré et de l'addition de bicarbonate de sodium. Ann. Zootech., 32, 81-92.

Dulphy J.P., Komar A., Zwaenepoel P., 1984. Effets comparés des traitements à l'ammoniac et à la soude sur la valeur alimentaire des fourrages pauvres. An. Zootech., 33, 321-342.

Dulphy J.P., Breton J., Bienaime A., Louyot J.M., 1982a. Etude sur la valeur alimentaire des pailles de céréales traitées ou non à la soude. I - Influence du traitement à la soude. Ann. Zootech., 31, 195-214.

Dulphy J.P., Bony J., Rouel J., Giraud J.M., 1986b. Utilisation de foins humides traités à l'ammoniac par des génisses. Bull. Techn. CRZV Theix, INRA, 66, 31-35.

Dulphy J.P., Kouassı A., Bienaime A., 1982b. Etude de la valeur alimentaire des pailles de céréales traitées ou non à la soude. II - Influence de la nature du complément énergétique. Ann. Zootech., 31, 215-232.

Gouet Ph., Grain J., Dubourguier H.C., Albagnac G., 1986. Interactions entre espèces microbiennes anaérobies dans le rumen. Reprod. Nutr. Dévelop., 26, 147-159.

Hemsley J.A., MoIr R.J., 1963. The influence of higher volatile fatty acids on the intake of ureasupplemented low quality cereal hay by sheep. Austr. I. Agri. Res., 14, 509-517.

INRA, 1978. Alimentation des Ruminants. INRA Publications, Route de Saint-Cyr, 78000 Versailles, $597 \mathrm{p}$.

JouANY J.P., 1981. Dosage des acides gras volatils et des alcools dans les ensilages par chromatographie en phase gazeuse. Bull. Tech. CRZV Theix, INRA, 46, 63-66.

Jung H.G., FAHEY G.C., 1981. Effect of phenolic compound removal on in vitro forage digestibility. J. Agric. Food. Chem., 29, n" 4, 817-820.

Laligant A., 1986. Etude des modifications biochimiques des pailles au cours d'un traitement par l'ammoniac. D.E.A. en Sciences Alimentaires. Université de Montpellier.

MAENG W.J., BALDWIN R.L., 1976. Factors influencing rumen microbial growth rates and yields: effect of amino-acids additions to a purified diet with nitrogen from urea. J. Dairy Sci., 59, 648-655.

Mir P.S., Mir Z., Robertson J.A., 1986. Effect of branched-chain amino acids or fatty acid supplementation on in vitro digestibility of barley straw or alfalfa hay. Can. J. Anim. Sci., 66, 151-156.

Nel.son M.L., Klopfensti:in T.J., Britton R.A., Lowry S.R., 1985. Protcin supplementation of ammoniated roughages. III - Corncobs supplemented with a blood meal-corn gluten meal mixture - Steer studies. J. Anim. Sci., 61, 1567-1575.

Tisserand J.L., Bellet B., Masson C., 1986. Effet du traitement des fourrages par la soude sur la composition de l'écosystème microbien du rumen des ovins et des caprins. Reprod. Nutr. Develop., 26 (1B), 313-314.

Tuah A.K., Tait R.M., 1985. Protein metabolism of sheep fed formaldehyde treated forage supplemented with iso-acids and sulfur. Nutr. Rep. Int., 32, $\mathrm{n}^{\circ}$ 4, 893-900).

VAREl V.H., JUNG H.G., 1986. Influence of forage phenolics on ruminal fibrolytic bacteria and in vitro fiber degradation. Appl. Environ. Microbiol., 52, 275-280.

ZwaEnePoel P., 1986. Biodétériorations et conservation des foins humides. Thèse de DocteurIngénieur. Université de Clermont II, $214 \mathrm{p}$. 